\title{
AIR FLOW MODELING IN DEEP WELLS: APPLICATION TO MINING VENTILATION
}

\author{
E. WITRANT ${ }^{1}$, K.H. JOHANSSON ${ }^{2}$ and the HynX team $^{3}{ }^{*}$, June 23, 2008
}

\begin{abstract}
In this paper, we present a novel air flow modeling strategy for deep wells that is suitable for real-time control of large-scale systems. We consider the mining ventilation control application, where specifically designed models are crucial for new automation strategies based on global system control and energy consumption optimization. Two different levels of complexity are proposed. Starting from a general model based on NavierStokes equations, we derive a $0-D$, Bond Graph model. This model is used to set a real-time simulator for the mine aerology problem.
\end{abstract}

\section{Introduction}

Traditionally, the control of large-scale systems, such as mining ventilation, has been performed locally with decentralized control actions. The terms control and optimization consequently refer to the preliminary design of the global system and automation devices. Mining ventilation provides for an interesting example of the automation evolution for large-scale systems. After a short bibliographical investigation, without any pretention of completeness, we can track mathematical modeling efforts back to 1968, where a steady-state compartmental model was proposed for flow networks with complex topology [1]. In 1973, new results on the experimental determination of turbulent diffusion coefficients were presented in [2], indicating a motivation for more advanced models. We have to wait until the 90's to find the first use of Navier-Stokes equations, with simplified chamber-like [3] and general mine aerology [4] models. The associated problems of nonlinearity and nonstationary behavior, high dimensionality and numerical issues have been quickly reported

\footnotetext{
*The authors are with 1 University Joseph Fourrier / GIPSA-lab, Grenoble, France, 2 KTH, Stockholm, Sweden, and 3 Cahors, France. The corresponding author email is emmanuel.witrant@gipsa-lab. inpg. fr.

This work has been supported in part by the European Commission through the projects HYCON and SOCRADES.
}

[5]. This short historical overview also illustrates the parallel evolution of magnetic ventilation modeling and computation capability.

Mining engineers have been primarily concerned with the proper design of ventilation tunnels, fans and turbines, and more generally the short and long term planning of ventilation requirements [6]. Recent technological developments, in the fields of embedded control and wireless sensing capabilities, motivate some new research efforts seeking global automation strategies that would allow for an optimized real-time control of the ventilation system. The novel socio-political concern for energy consumption and increase of electricity prices in a context of worldwide competition also motivate theoretical research leading to improved control algorithms.

In this paper, we address the problem of defining a proper physical model of the air flow in a deep well with point-wise exhausts for real-time control applications. More precisely, we define a novel model for the main ventilation shaft, extraction rooms and exhaust, where the aerodynamics depends on the atmospheric conditions, surface heater and turbine, underground fans operation and extraction rooms flow conditions. Starting from the full Navier-Stokes equations (conservation of mass, momentum and energy) a 0-D model, based on a bond-graph approach, is proposed to model the full mine aerology problem. This model includes the main aerodynamics and has the necessary real-time capabilities for future developments of model-based control strategies, as illustrated by the simulations.

The paper is organized as follows. First, the mining ventilation problem is described and the main physical properties are provided. A 2-D model based on NavierStokes equations is presented in Section 3. The bondgraph 0-D model is addressed in Section 4 and followed by simulations results that detail the dynamics of the flow for a specific test-case. 


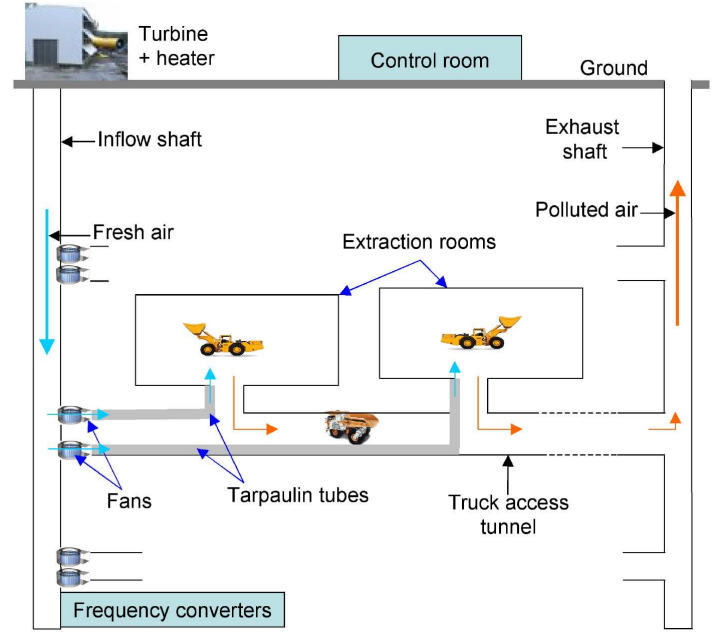

Figure 1. Airflows in an underground mine.

\section{Mining ventilation description}

The mine ventilation topology considered in this work is presented in Figure 1, where fresh air is carried from the surface to the extraction rooms through a vertical inflow shaft and is expelled through an exhaust shaft. From a control perspective, this ventilation system can be divided into two subsystems:

- the primary system, which corresponds to the inflow shaft and associated automation devices. Its dynamics is governed by the Navier-Stokes equations (including the compressibility, viscous losses and thermodynamic effects), due to the large mass flow rate and the length of the shaft considered. The regulated inputs are the heater (used during winter in northern countries to prevent freezing in the shaft's entrance) and turbine powers. The fans as well as atmospheric conditions act as known disturbances on this system. The primary system provides the fans with an appropriate flow pressure, which is then the regulated output;

- the secondary systems, which include the airflows between the fans (included) and the extraction rooms exits. Note that there is one secondary system for each extraction level. For this system, the aerodynamics of the flow can be greatly simplified in comparison with the uncertainties induced by the complexity of the chemical processes. We then consider the airflow in the tarpaulin tubes as inviscid, incompressible and adiabatic, and use a semi-empirical model for the chemical processes that strongly relies on the use of a wireless sensor network in the extraction room. The secondary system is actuated by the fans, the regulated output is the gas concentration in the room and the vertical shaft pressure as well as the number of trucks in the room are known disturbances.

The interconnection between the two subsystems is the pressure at the fans location, which is a key parameter to investigate the relative efficiency of the turbine with respect to the fans to ensure that the room gas concentration is within safety bounds.

The global modeling and control problem is then divided into two subproblems:

- one that is driven by continuous dynamics and for which we can obtain a precise model from physical laws (inflow shaft);

- one with hybrid dynamics (the discrete part being the number of trucks in the room) and a highly uncertain model, thus strongly relying on the available measurements (secondary systems).

Detailed modeling and control of the secondary system is addressed in the companion papers $[8,9]$. The present paper is focused on the aerodynamics modeling in the ventilation shafts, highlighting the behavior of a large airflow in a deep well, and the secondary systems are considered as smaller tubes with an expansion at the extraction rooms locations.

\section{Navier-Stokes 2-D model}

We consider the compressible and viscous laminar flow in the vertical ventilation shaft presented in the left part of Fig. 2(a), where $x$ is the vertical direction (with $x=0$ at the ground level and $x=L$ at the bottom of the shaft) and $y$ indicates the position along the diameter. This flow is characterized by its density $\rho(x, y, t)$, velocity $\mathbf{V}(x, y, t)=u(x, y, t) \mathbf{i}+v(x, y, t) \mathbf{j}$ (where $\mathbf{i}$ and $\mathbf{j}$ are unit vectors in the $x$ and $y$ directions), pressure $p(x, y, t)$ and temperature $T(x, y, t)$.

The dynamics of the flow is obtained from the conservation of mass, momentum and energy, along with the perfect gas equation of state $p=\rho R T$, where $R$ is the specific gas constant, and the hypothesis that the gas is calorifically perfect $e=c_{v} T$, where $c_{v}=R /(\gamma-1)$ $(\gamma=1.4)$ is the specific heat at constant volume. Choosing a conservative form (in the numerical sense) of Navier-Stokes equations, we have that [10]:

$$
\frac{\partial}{\partial t}\left[\begin{array}{c}
\rho \\
\rho \mathbf{V} \\
\rho E
\end{array}\right]+\nabla \cdot\left[\begin{array}{c}
\rho \mathbf{V} \\
\rho \mathbf{V}^{T} \otimes \mathbf{V}+p \mathbf{I}-\tau \\
\rho \mathbf{V} H-\tau \cdot \mathbf{V}-k \nabla T
\end{array}\right]=\left[\begin{array}{c}
0 \\
0 \\
\dot{q}
\end{array}\right]
$$

where $\otimes$ is the tensor product of two vectors, $\tau$ is the shear stress vector, $k(x, y, t)$ is the thermal conductivity, 


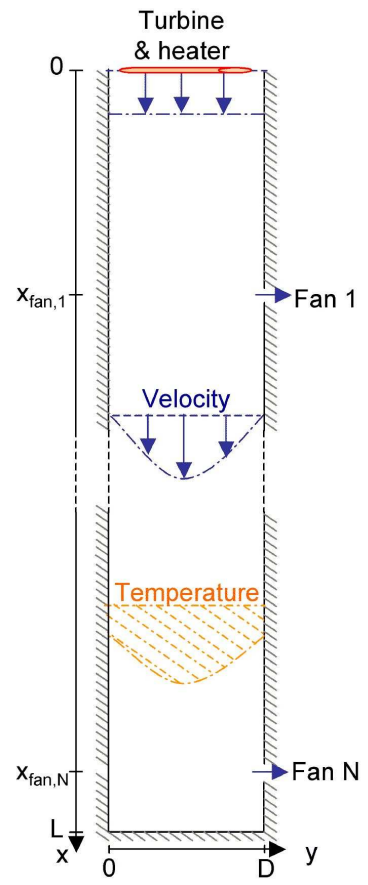

(a) 2D model

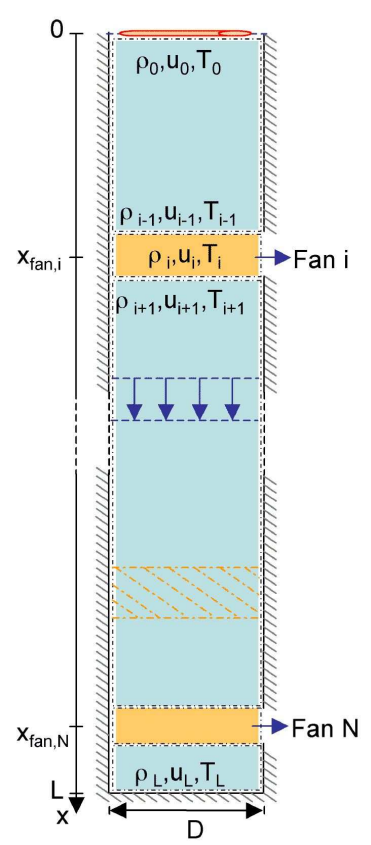

(b) Averaged model
Figure 2. Vertical shaft aerodynamics: 2-D model and volume-averaged approximation.

$H=E+p / \rho$ is the total enthalpy, $E$ is the total energy per unit mass and $\dot{q}(x, y, t)$ is the rate of heat addition (see [11] for a precise description). Using the subscripts to indicate the partial derivatives dependency, $\tau$ is computed from

$$
\left[\begin{array}{c}
\tau_{x x} \\
\tau_{x y} \\
\tau_{y y}
\end{array}\right]=\left[\begin{array}{c}
\lambda \\
\mu \\
\lambda
\end{array}\right](\nabla \cdot \mathbf{V})+2 \mu\left[\begin{array}{c}
u_{x} \\
0 \\
v_{y}
\end{array}\right]
$$

where $\mu(x, y, t)$ is the viscosity and $\lambda(x, y, t)$ is the bulk viscosity coefficient. They can be computed using Stokes and Sutherland approximations as

$$
\lambda=-\frac{2}{3} \mu \quad \text { and } \quad \frac{\mu}{\mu_{s l}}=\left(\frac{T}{T_{s l}}\right)^{3 / 2} \frac{T_{s l}+110}{T+110}
$$

where the $s l$ subscript denotes the sea level conditions. The thermal conductivity is obtained from $k=$ $1.45 \mu c_{p}$, where $c_{p}=\gamma R /(\gamma-1)$ is the specific heat at constant pressure.

Another possibility is to use the experimental results presented in [12], where turbulent diffusion and heat conduction coefficients in underground mines are proposed. Such model can be adapted to the set of equations (1) thanks to a proper definition of the shear stress vector $\tau$.
As we are considering a viscous flow, specific care should be taken in the definition of the boundary conditions (BC). The inflow BC are the same as in the inviscid case but the wall conditions are greatly modified (no-slip BC on velocity and temperature status set by the geothermal effect). The outflows BC are also critical, as the fans operation may destabilize the numerical scheme. The presence of boundary layers implies a particularly refined mesh close to the walls.

Arbitrary initial conditions (i.e. the sea level ones) and a classical discretization scheme, such as MacCormac's predictor-corrector method, can be used for simulation purposes.

\section{Bond graph 0-D model}

Non-dimensional modeling has an increasing use in the design, validation and tuning of control laws. The two main advantages of this approach are the integration of as many physical properties as possible (avoid data mapping) and a reduced computation time (close to real-time, approximately 10 times slower in the worst cases). It then allows for predictive models on nonidentified operating points with satisfying computation times. The model components presented here are directly inferred from $[13,14]$ and based on 1D Euler equations (inviscid flow hypothesis). This section is focused on the main dynamics of the model and its application to the mining case, leading to the bond graph representation. We refer to [14] for a more detailed description.

This model is based on Euler equation by considering the time differential equation

$$
\frac{\partial}{\partial t}\left[\begin{array}{c}
\rho \\
\rho \mathbf{V} \\
\rho E
\end{array}\right]+\nabla \cdot\left[\begin{array}{c}
\rho \mathbf{V} \\
\rho \mathbf{V}^{T} \otimes \mathbf{V}+p \mathbf{I} \\
\rho \mathbf{V} H
\end{array}\right]=\left[\begin{array}{l}
0 \\
0 \\
0
\end{array}\right]
$$

which is discretized using finite volume method. The flow dynamics in a given control volume are directly obtained by considering time differential operator $\partial \cdot / \partial t$ as the source of internal flow dynamics and the gradient operator $\nabla$ as the difference between output and input flow conditions (see [13] for more details). The resulting dynamics are simplified thanks to the hypotheses:

H1) Only the static pressure is considered, implying that the kinetic energy term in the energy conservation equation is omitted ( $E=U$, the internal energy);

H2) The impulsive term is negligible compared to the pressure: $\rho v^{2}<<p$ in the momentum conservation equation. 
The conservation equations are then written as

$$
\frac{d}{d t}\left[\begin{array}{c}
m \\
U \\
Q_{m}
\end{array}\right]=\left[\begin{array}{c}
Q_{m, i}-Q_{m, o} \\
Q_{m, i} h_{i}-Q_{m, o} h_{o} \\
\frac{S}{L}\left(p_{i}-p_{o}\right)
\end{array}\right]
$$

where $m$ is the mass, $Q_{m}(t)=\rho S v=L / S \int_{0}^{t} \Delta p(s) d s$ the mass flow, $h=c_{p} T$ the mass enthalpy, $S$ the flow cross section and $L$ the control volume length. The subscripts $i$ and $o$ denotes the input and output components, respectively.

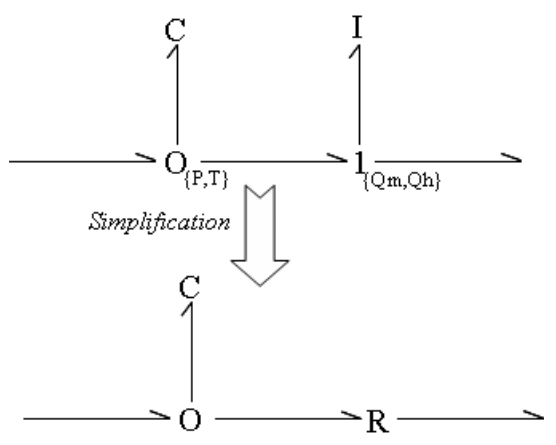

Figure 3. Bond graph model of the control volume.

Note that the enthalpy flow is directly obtained as $Q_{h}=Q_{m} c_{p} T$. The pressure and temperature are obtained from the state variables using the first Joule law $\left(U=m c_{v} T\right)$ and the perfect gas relationship as

$$
T=\frac{\gamma-1}{R} \frac{U}{m} \quad \text { and } \quad p=(\gamma-1) \frac{U}{v}
$$

Pressure losses are typically linear or singular, and modelled thanks to Bernoulli's equation (supposing incompressibility) as $\Delta P=\zeta Q_{m}^{2} /\left(2 \rho S^{2}\right)$, where $\zeta$ is a friction coefficient.

Bond graph description (see Fig. 3) can now be introduced with capacitive $\mathbf{C}$ (mass and energy conservation), inductive $\mathbf{I}$ (momentum equation) and resistive $\mathbf{R}$ elements. The efforts are the pressure and temperature, and the flows are the mass and enthalpy. The resistive elements are introduced to approximate the momentum equation with the algebraic Saint Venant relationship

$$
Q_{m}=\rho C_{d} S \sqrt{\gamma R T_{i}}\left(\frac{2}{\gamma+1}\right)^{\frac{\gamma+1}{2(\gamma-1)}}
$$

where $C_{d}$ is an experimentally-based correction factor. This approximation reduces the numerical instabilities introduced by the inductive term and allows for a larger computation time-step.

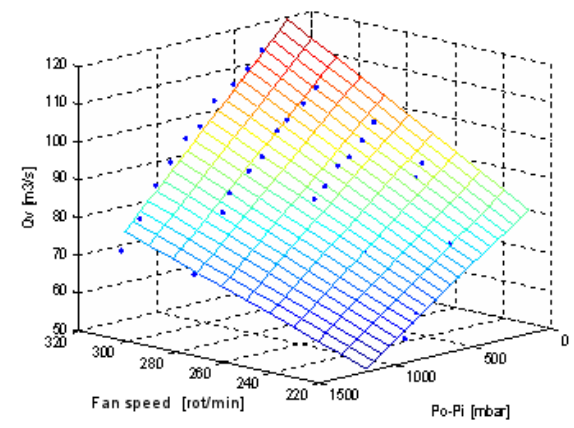

Figure 4. Fan specification map (blue points: experimental values).

The turbine and fans are compressors that generate a flow depending on a pressure gradient and a rotational speed. Their characteristics depend on specification maps such as the one presented in Fig. 4. The fans enthalpic flow simply write as $Q_{h}=Q_{m} c_{p} T$. Supposing adiabatic and isentropic compression, the output temperature is obtained as

$$
T_{o}=T_{i}\left\{\frac{1}{\eta_{c}}\left[\left(\frac{P_{o}}{P_{i}}\right)^{\frac{\gamma-1}{\gamma}}-1\right]+1\right\}
$$

where $\eta_{c}$ is a conversion efficiency factor. The bond graph model is finally constructed from the previous effort and flow relationships, using (4) and (5) for the capacitive and resistive elements, respectively.

\section{Simulation results}

The inflow and outflow ventilation shafts are both discretized with 28 control volumes. The extraction levels ( 3 in this simulation) are modelled with a room and two control volumes on each side, as presented in Fig. 5. Heat exchange is introduced, in each control volume, to take into account the geothermal effect. This is modelled with $Q_{p}=H_{c} S \Delta T$, where $H_{c}$ depends on air temperature and flow. The resulting simulator, coded in Simulink ${ }^{\circledR}$, allows for the regulation of the turbine and fans. Flows, pressures and temperatures in each control volume can be measured. The case study presented here corresponds to the following scenario: the turbine rotation speed is increased from 260 to $280 \mathrm{rpm}$ at $t=1000 \mathrm{~s}$, the $1^{s t}$ level fan is not operated, the $2^{\text {nd }}$ level fan goes from 0 to $150 \mathrm{rpm}$ at $t=2000 \mathrm{~s}$ and the $3^{\text {rd }}$ level fan is operated at $200 \mathrm{rpm}$ during the whole simulation. A $C O$ pollution is injected at $t=500 \mathrm{~s}$ during $80 s$ in the $3^{r d}$ level room. The time evolution of the 


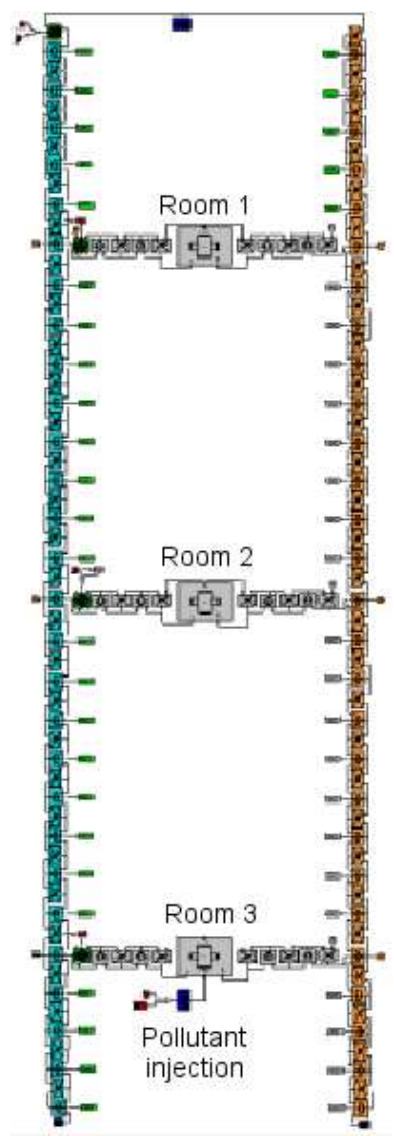

Figure 5. HynX OD model of the mine ventilation. flow, pressure, temperature and pollution is measured at the surface and extraction levels.

The simulation results presented in Fig. 6 illustrate the time evolution of the physical and chemical airflow properties for the scenario described above at different depths. The pressure losses in the inflow shaft (Fig. 6(a)) show the energy losses associated with the flow transport in a deep well, the increase associated with the turbine extra-actuation and the drastic pressure losses that occur when the fan 2 is switched on. The rooms ventilation rate (Fig. 6(b)) highlight the physical interconnection between the rooms and the importance of a global control strategy for the primary system.

The temperature evolution (Fig. 6(c), where we chose a particularly cold initial condition to emphasize the dynamics) is driven by the geothermal effect and strongly affected by the fans compression. We are also able to take into account $N O_{x}$ and/or $C O$ pollutant and track the concentration evolution along the exhaust shaft (Fig. 6(d)): the time-delay effect associated with the transport phenomenon is particularly highlighted. The computation time is very satisfying as the simulation is 34 times faster than real-time in an Intel Centrino ${ }^{\circledR} 1.83 \mathrm{GHz}$ PC.

The proposed 0-D model efficiently provides for a fast and representative global model for the mine ventilation problem. Indeed, a good quantitative estimation of the main dynamics and associated delays is obtained despite the relatively low number of states. The precision can be increased by increasing the number of control volumes and the associated number of state variables, but it has a direct consequence on the simulation time. The proposed simulation is based on 142 state variables ( 28 cells per shaft, 5 cells per extraction level and 2 states per cell) and is 34 times faster than realtime, which is particularly appropriate for the design of control laws.

\section{Conclusions}

In this work, we addressed the problem of providing an appropriate model for mining ventilation control as a modeling problem focused on the flow aerodynamics. Two different classes of models were considered: the classical 2-D Navier-Stokes equation and a 0 -D bond-graph model. Several issues, such as turbulence, nonlinearities and interconnections, were briefly discussed. The main objective of theses models is to motivate future works on model-based real-time control (simulation results of the 0-D model illustrate its usefulness for such applications), advanced PDE-based control strategies (1-D model) or the development of complex simulators for flows analysis (2-D model). 


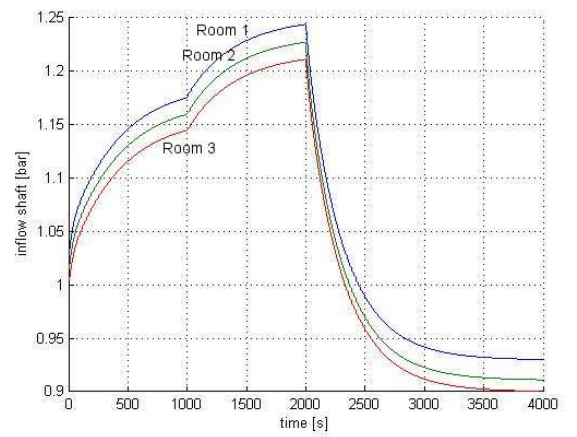

(a) Pressure losses in the inflow shaft

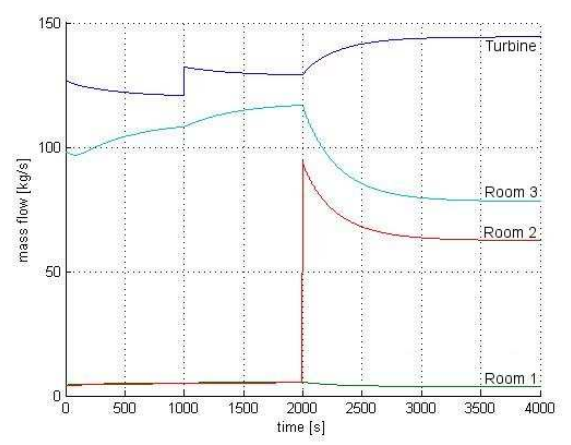

(b) Extraction rooms ventilation rate

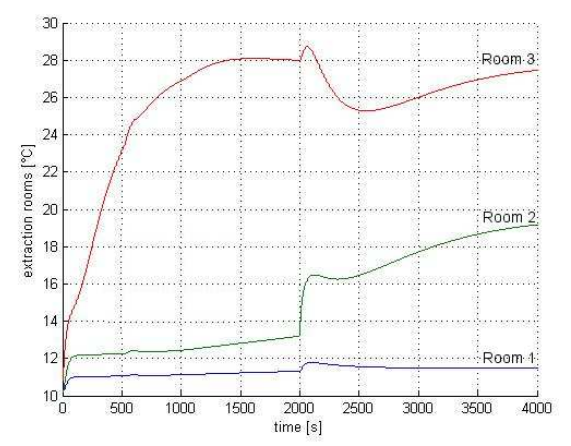

(c) Extraction rooms temperature

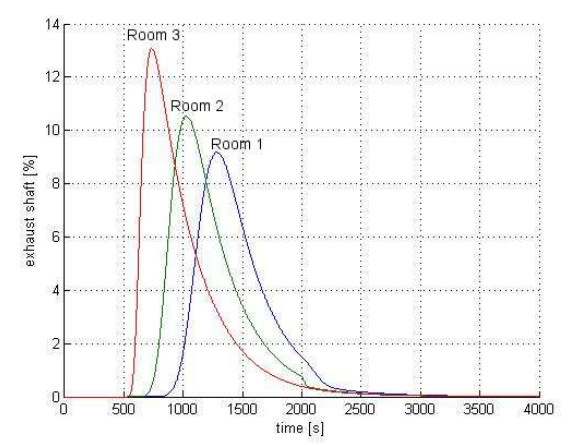

(d) CO pollutant concentrations in the exhaust shaft

\section{References}

[1] S. Tolmachev and E. Fainshtein, "Analysis of multibranch junctions in ventilation networks, regarded as systems with distributed parameters," Journal of Mining Science, vol. 4(2), pp. 128-133, 1968.

[2] F. Klebanov and G. Martynyuk, "A method for experimental determination of the coefficient of longitudinal turbulent diffusion in ventilating currents of mine workings," Journal of Mining Science, vol. 9(4), pp. 413-416, 1973.

[3] G. Kalabin, A. Baklanov, and P. Amosov, "Calculating the aerogas dynamics of chamber-like mine workings on the basis of mathematical modeling," Journal of Mining Science, vol. 26(01), pp. 61-73, 1990.

[4] N. Petrov, M. Shishkin, V. Dnitriev, and V. Shadrin, "Modeling mine aerology problems," Journal of Mining Science, vol. 28(2), pp. 185-191, 1992.

[5] N. Petrov, "Methods of solving problems and designing equipment for mine ventilation," Journal of Mining Science, vol. 30(2), pp. 195-204, 1994.

[6] E. Widzyk-Capehart and B. Watson, "Agnew gold mine expansion mine ventilation evaluation using VentSim," in Proc. of the $7^{\text {th }}$ International Mine Ventilation Congress, 2001.

[7] S. Olaru, G. Sandou, E. Witrant, and S. Niculescu, "Receding horizon climate control in metal mine extraction rooms," in Submitted to IEEE CASE'08, 2008.

[8] M. Di Benedetto, A. D'Innocenzo, E. Serra, and E. Witrant, "Automatic verification of wireless control in a mining ventilation system," in Submitted to IEEE CASE'08, 2008.

[9] C. Hirsch, Numerical Computation of Internal \& External Flows: the Fundamentals of Computational Fluid Dynamics, $2^{\text {nd }}$ ed. Butterworth-Heinemann (Elsevier), 2007.

[10] J. Brown, A. Vardy, and A. Tijsseling, "Response of wall heat transfer to flows along a cylindrical cavity and to seepage flows in the surrounding medium," Eindhoven: Technische Universiteit Eindhoven, Tech. Rep., 2005. [Online]. Available: ftp://ftp.win.tue.nl/ pub/rana/rana05-35.pdf

[11] G. Averin and A. Yakovenko, "Determination of the turbulent diffusion and heat-conduction coefficients in mine workings," Journal of Mining Science, vol. 26(5), pp. 465-467, 1990.

[12] V. Talon, "Modélisation 0-1 d des moteurs à allumage commandé," Ph.D. dissertation, Université d'Orléans, 2004.

[13] V. Talon and S. Cstric, "Engine control model based design with achille library," E-COM: Rencontres scientifiques de l'IFP, pp. 33-51, Oct. 2006.

\section{Figure 6. Simulation results for a 3-levels mine.}

